\title{
A Node Reputation Evaluation Model for Mobile P2P
}

\author{
Meijuan Jia \\ College of Computer Science and Technology \\ Harbin Engineering University \\ Harbin, China \\ College of Computer Science and Information Technology \\ Daqing Normal University \\ Daqing, China \\ e-mail: jiameijuan@sina.com \\ Huiqiang Wang \\ College of Computer Science and Technology \\ Harbin Engineering University \\ Harbin, China \\ e-mail: wanghuiqiang@hrbeu.edu.cn
}

\author{
Guangsheng Feng \\ College of Computer Science and Technology \\ Harbin Engineering University \\ Harbin, Chi \\ e-mail: fengguangsheng@hrbeu.edu.cn \\ Fangfang Guo \\ College of Computer Science and Technology \\ Harbin Engineering University \\ Harbin, China \\ e-mail:guofangfang@hrbeu.edu.cn
}

\begin{abstract}
The characteristics of dynamic, heterogeneity and limited resources of nodes result in the existence of selfish behavior of nodes in MP2P network, which affect the network performance. To improve the performance of MP2P, an evaluation model for node reputation is established by combining the analytic hierarchy process with the fuzzy comprehensive evaluation technology in this paper. Direct trust, indirect trust, residual energy and active degree of nodes are used as the fundamental variables and their weights are determined by using analytic hierarchy process. And then the reputation of nodes is calculated using membership function and fuzzy comprehensive evaluation method. Nodes with high reputation are allowed to participate in the communication and nodes with low reputation are not allowed. The experimental simulation results show that our model can not only effectively improve the delivery ratio of packet but also reduce the average latency and overload ratio of the network.
\end{abstract}

Keywords- MP2P network; trust; reputation evaluation model; analytic hierarchy process; fuzzy comprehensive evaluation

\section{INTRODUCTION}

Over the last decade, Peer-to-Peer (P2P) systems have become very popular, and have been used to provide solutions in areas ${ }^{[1]}$. P2P architectures will be very important for future distributed systems and applications. In such networks, peers are heterogeneous in providing services but they do not have the same competence of reliability. Therefore, it is necessary to estimate whether a peer is trustworthy or not for file sharing and other services. In the process of communication, the future behavior of nodes can be judged according to the performance and the characteristics, which is known as nodes' reputation. We can calculate nodes' reputation based on nodes' reputation mechanism, which is used as the basis of routing ${ }^{[2]}$. Many researchers have proposed some related frameworks and solutions around nodes' reputation evaluation mechanism. The method proposed addresses a super-peer based trust model for Peer-to-Peer (P2P) networks to solve the problems that the trust relation between peers is not sufficiently built due to the difference of peers' interests and low probability of repeated transactions between them ${ }^{[3]}$. An extension to an existing ad hoc routing protocol has been proposed ${ }^{[4]}$. This method selects the route based on a local evaluation of the trustworthiness of all known intermediary nodes (routers) on the route to the destination. It has showed that how trust can be built from previous experience and how trust can be used to avoid unreliable nodes during the routing process. To motivate the excessive selfish nodes to actively cooperate with other nodes, an incentive and cooperation model based on punishment mechanism is presented ${ }^{[5]}$. Manickam et al. proposed a fuzzy based ad hoc on-demand distance vector routine protocol for MANET ${ }^{[6]}$. It can evaluate nodes' reputation using fuzzy logic, set threshold and nodes' selfish behavior. The author presents an integrated fuzzy-based trustworthiness system for communications in JXTA-overlay P2P platform ${ }^{[7]}$. This system consists of two Fuzzy Logic Controllers (FLC1 and FLC2). FLC1 has three input parameters: namely Number of Jobs (NJ), Number of Connections (NC) and Connection Lifetime (CL) and its output is Actual Behavioral Criterion (ABC). Then ABC and Reputation (R) are used as input systems for FLC2 and the corresponding output is Peer Reliability (PR).

In this paper, a node reputation fuzzy comprehensive evaluation model is proposed. The weights of direct trust, indirect trust, residual energy and active degree of nodes are determined, and the nodes' reputation can confirm according to the membership function and fuzzy comprehensive evaluation method.

\section{PROPOSED NODES REPUTATION FUZZY EVALUATION MODEL}

To construct the model, we make some necessary assumptions and definitions prior to the concrete analysis. 
Assumption 1 There exist no selfish nodes in the network, in other words, nodes will not report false information to others, and there exist no collusion between nodes ${ }^{[8]}$.

Assumption 2 Nodes are in promiscuous mode when they are in the network's operation, namely, all nodes can monitor the information of other nodes within the range of the transmission.

Assumption 3 Network is composed of isomorphic nodes. Each node in the network has the same computing power, storage capacity, communication distance and the initial energy.

Assumption 4 A node can't complement energy for each other, it will leave from the network while energy exhausts.

The model of node reputation evaluation is shown in Fig.1.
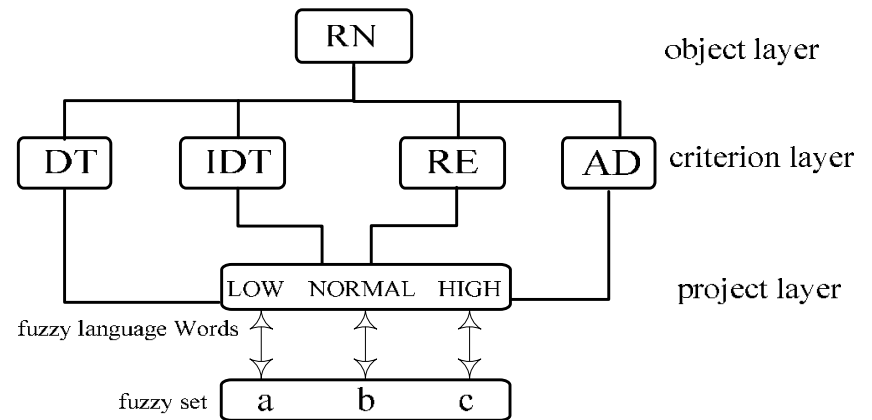

Figure 1 Fuzzy Evaluation model of Node Reputation

Direct trust (DT) is estimated by the node's physical neighbors based on its real-time forwarding ratio, which is denoted by $D T\left(n_{i}, n_{j}\right)$, and $0 \leq D T\left(n_{i}, n_{j}\right) \leq 1$. As shown in Fig.2.

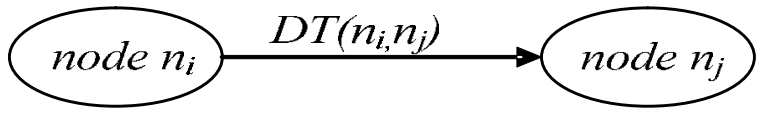

Figure 2 Direct Trust model

Indirect Trust (IDT) is evaluated by the weighted average of DT which is provided by $n_{i}$ 's neighbors interacting with $n_{j}$. It is denoted by $\operatorname{IDT}\left(n_{i}, n_{j}\right)$, as shown in Fig.3. Thus $\operatorname{IDT}\left(n_{i}, n_{j}\right)$ is defined as follows:

$$
\operatorname{IDT}\left(n_{i}, n_{j}\right)=\frac{1}{n} \sum_{m=1}^{n} W_{i}^{m} * D T\left(\text { neig }_{i}{ }^{m}, n_{j}\right)
$$

Where $\operatorname{IDT}\left(n_{i}, n_{j}\right)$ is indirect trust that node $n_{i}$ has about node $n_{j}$. The neighbor number of node $n_{i}$ is denoted by $n$, the $m^{\text {th }}$ neighbor of node $n_{i}$ is denoted by $n e i g_{i}^{m}$. The weight of the $m^{\text {th }}$ neighbor of node $n_{i}$ is denoted by $W_{i}^{m}$, which can be computed by:

$$
W_{i}^{m}=\frac{D T\left(n_{i}, n e i g_{i}^{m}\right)}{\sum_{m=1}^{n} D T\left(n_{i}, n e i g_{i}^{m}\right)}
$$

The direct trust that node $n_{i}$ has about node $n^{2} i g_{i}^{m}$ is denoted by $D T\left(n_{i}, n e i g_{i}^{m}\right)$.

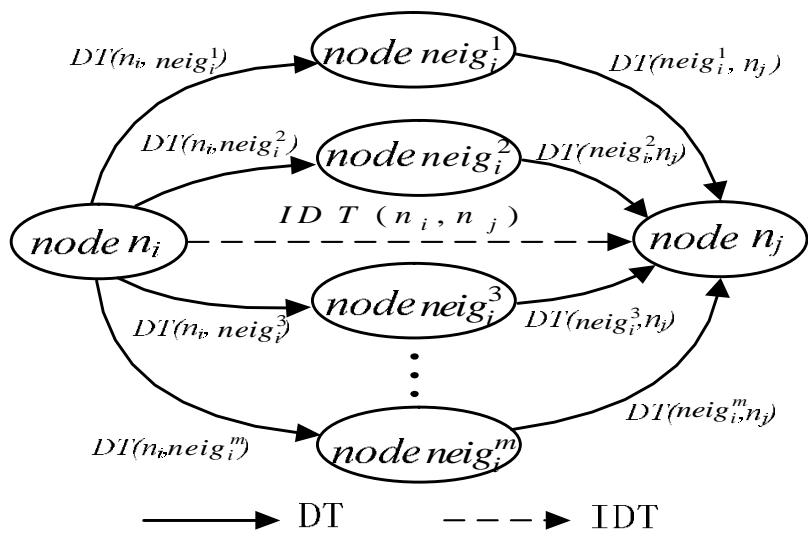

Figure 3 Indirect Trust model

In the process of communication, each node will lose its energy because of providing service for others. The more the node involved in communications, the more energy will be consumed ${ }^{[9]}$. Residual energy is denoted by $R E_{i}$. The cumulative number of entities interacting with an evaluated node $n_{i}$, which is denoted by $A D_{i} . R N_{i}$ denotes node $n_{i}{ }^{\prime} s$ trust level, which can be computed by :

$$
\begin{aligned}
R N_{i} & =\omega_{1} * D T\left(n_{i}, n_{j}\right)+\omega_{2} * \operatorname{IDT}\left(n_{i}, n_{j}\right) \\
& +\omega_{3} * R E_{i}+\omega_{4} * A D_{i}
\end{aligned}
$$

Where $\omega_{i}$ is the weight of four factors, $1 \leq i \leq 4$, $0 \leq \omega_{i} \leq 1$ and $\sum_{i=1}^{4} \omega_{i}=1$.

\section{CAlCulate the Reputation}

Decision making is very difficult in vague and uncertain environments. This vagueness and uncertainty can be handled using fuzzy comprehensive evaluation ${ }^{[10]}$, because it not only can establish relationship between qualitative analysis and quantitative analysis, but also has strong ability of comprehensive judgment.

Step 1: Confirm comment set

According to the decision factors, we confirm the comment set:

$$
V=\left\{\text { low }\left(v_{1}\right), \text { normal }\left(v_{2}\right), \text { high }\left(v_{3}\right)\right\}
$$

Step 2: Construct the fuzzy comparison matrix

By using TFN ${ }^{[11]}$, the decision factors are required to make pairwise comparisons for the main criteria and sub criteria. A fuzzy comparison matrix $A$ is constructed according to arithmetic mean of pairwise comparisons from decision factors.

Step 3: Determine the weight of factors

The weight of each factor will be determined by normalizing any of the rows or columns of matrix $A$. By calculating the maximum eigenvalue of the matrix using characteristic root method, we can get $\lambda_{\max }=4.209$. After 
normalization, the weight is $\omega_{1}=0.56, \omega_{2}=0.264$, $\omega_{3}=0.122, \quad \omega_{4}=0.054$.

Step 4: Check consistency

The consistency ratio (CR) is calculated in order to control the results of this method. The consistency can be checked by $\mathrm{CR}$, which is used to directly estimate the consistency of pairwise comparisons. CR is computed using equation (4).

$$
\begin{aligned}
& C R=\frac{C I}{R I} \\
& C I=\frac{\lambda_{\text {max }}-n}{n-1}
\end{aligned}
$$

Where $\mathrm{CI}$ is consistency index. RI is random index, and $\mathrm{n}$ is matrix size $\mathrm{s}^{[8]}$.

When $n=4, \mathrm{RI}=0.89^{[8]}$. So we can get :

$$
\begin{gathered}
C I=\frac{\lambda_{\max }-n}{n-1}=\frac{4.209-4}{4-1}=0.0697 \\
C R=\frac{C I}{R I}=\frac{0.0697}{0.89}=0.0783<0.1
\end{gathered}
$$

Step 5: Evaluate the reputation of nodes

Weighted average method (WAM) is very suitable for solving trust evaluation ${ }^{[12]}$, since it is easy to understand and implement. In this paper, with the established factor set, evaluation set, membership degree and weight set, we can get the result of fuzzy comprehensive evaluation using WAM and fuzzy arithmetic operators. The Fuzzy Comprehensive Evaluation (FCE) formula is $R N=W \mathrm{O} R$, which is expressed by vector form as follows:

$$
\left(r n_{1}, r n_{2}, \ldots, r n_{n}\right)=\left(\omega_{1}, \omega_{2}, \ldots, \omega_{n}\right) \circ\left(r_{i j}\right)_{n \times l}
$$

\section{SimUlations AND ANALYSIS}

NS-2 simulator is used to evaluate the performance of reactive routine protocols in different conditions. Within a rectangular field $1000 \mathrm{~m} \times 1000 \mathrm{~m}$, 50 nodes are randomly dispersed and the transmission radius in one hop is fixed at $250 \mathrm{~m}$. The node mobility uses the random waypoint model in

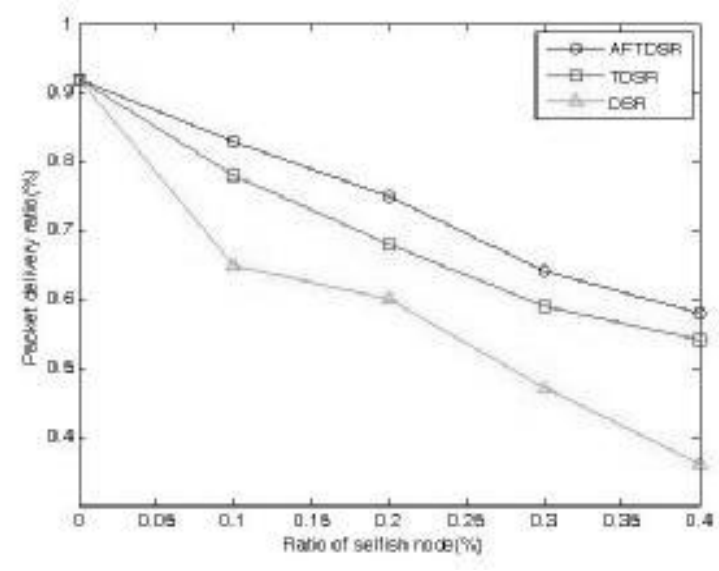

4 (a) Packet delivery ratio which each packet starts its journey from a location to another at a random speed. There are twenty selfish nodes in default. Selfish nodes will discard the packets from others. The fixed simulation parameters in NS-2 are listed in Table 1.

TABLE I . FIXED SIMULATION PARAMETERS

\begin{tabular}{|c|c|}
\hline Parameter & Value \\
\hline MAC & 802.11 \\
\hline Area & $1000 \mathrm{~m} \times 1000 \mathrm{~m}$ \\
\hline Transmission radius & $250 \mathrm{~m}$ \\
\hline Maximum node speed & $20 \mathrm{~m} / \mathrm{s}$ \\
\hline Placement & Uniform \\
\hline Movement model & Random waypoint \\
\hline Sending capacity & $2 \mathrm{Mbps}$ \\
\hline Stimulation time & $500 \mathrm{~s}$ \\
\hline
\end{tabular}

Selfish nodes will discard the packets from others. To evaluate the performance of AFTDSR, we compare AFTDSR (the protocol in this paper, AHP Fuzzy Trust DSR) with DSR and $\operatorname{TDSR}^{[7]}$.

When there are no selfish nodes, the packet loss ratio is about 8\%. As shown in Fig.4 (a), the delivery ratio in DSR degrades sharply while the ratios in TDSR and AFTDSR degrade gently, and the delivery ratios of TDSR and AFTDSR are always higher than that of DSR. As shown in Fig.4 (b), the average latency in TDSR and AFTDSR increases slowly with the increase ratio of selfish nodes. Fig.4 (c) shows the comparison of overload ratio for AFTDSR, TDSR and DSR. AFTDSR has the highest overload ratio as the ratio of selfish nodes increasing.

As shown in Fig.5 (a), with the increase of node speed, the delivery ratio of AFTDSR and TDSR gently declines while DSR decline remarkably. And the average latencies in DSR, TDSR and AFTDSR increase, as shown in Fig.5 (b). Fig.5 (c) illustrates that, with the increase of node speed, overload ratio in TDSR and AFTDSR remains comparatively higher than DSR.

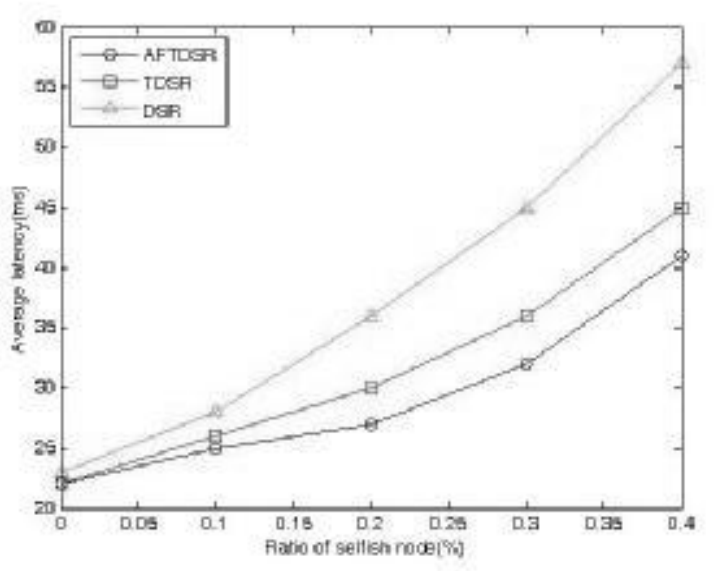

4 (b) Average latency 


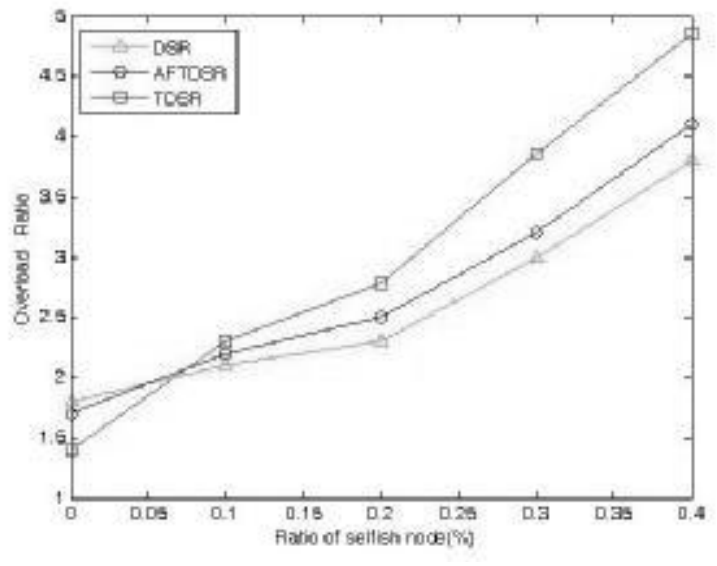

4 (c) Overload ratio

Figure 4. Comparison of network performance under different ratio of selfish node

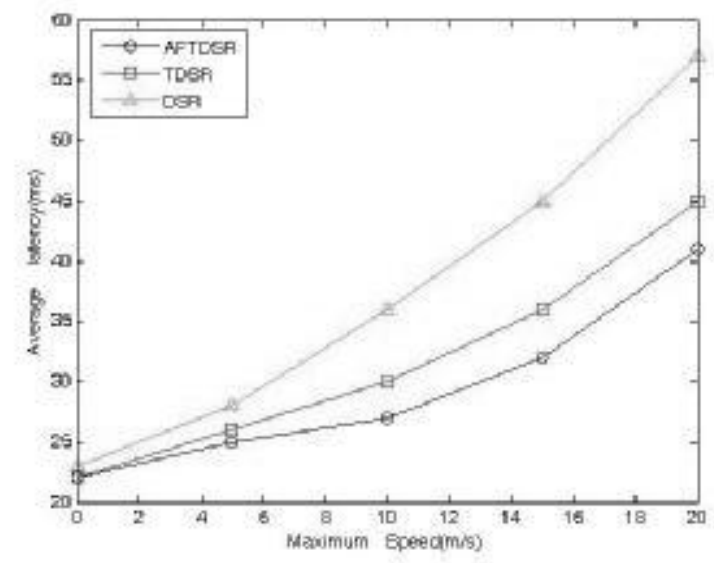

5 (b) Average latency

\section{CONCLUSIONS}

In this paper, we present a novel model to evaluate nodes reputation for MP2P by combining the analytic hierarchy process with the fuzzy comprehensive evaluation technology. Base on the model, we proposed a protocol which is extended from Source Routing Mechanism, called AFTDSR. Theperformance comparison of these routing protocols (DSR, TDSR and AFTDSR) shows that AFTDSR is able to achieve a remarkable improvement in the packet delivery ratio, average latency and overload ratio. In the future research, we will consider the collusion between nodes when there exist selfish nodes in the network.

\section{ACKNOWLEDGMENT}

This wok was supported by the National Natural Science Foundation of China (61370212), the Research Fund for the Doctoral Program of Higher Education of China (20122304130002), the Natural Science Foundation of Heilongjiang Province (ZD 201102), the Fundamental Research Fund for the Central Universities (HEUCFZ1213, HEUCF100601).

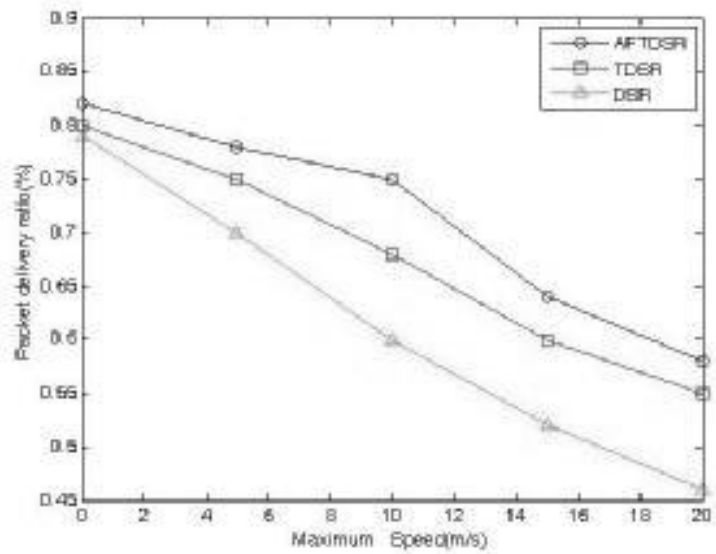

5 (a) Packet delivery ratio

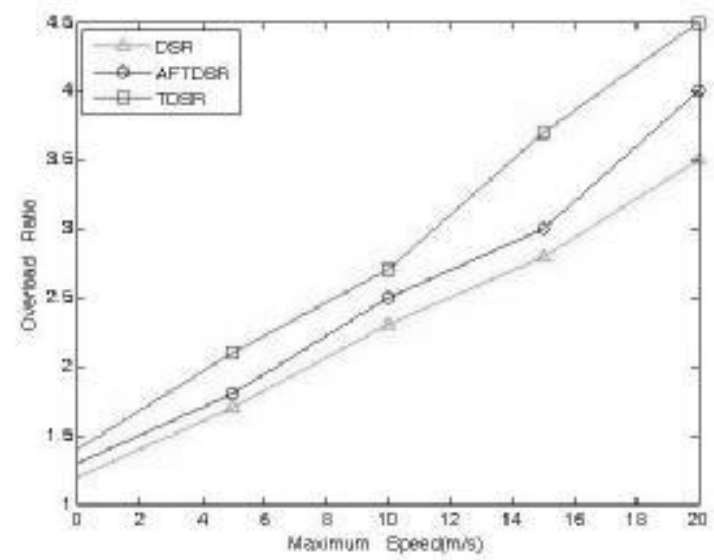

5 (c) Overload ratio

Figure 5. Comparison of network performance at different maximum node speed

\section{REFERENCES}

[1] Ou Zhong-hong, Song Mei-na, Zhan Xiao-su, et al. Key techniques for mobile peer-to-peer networks [J]. Journal of Software, 2008,19(2): 404-418.

[2] Josang A, Ismail R, Boyd C. A survey of trust and reputation systems for online service provision[J]. Decision Support Systems, 2007, 43(2):618-644.

[3] Tian Chun-qi, Jiang Jian-hui, Hu Zhi-guo, et al. A novel super-peer based trust model for peer-to-peer networks[J]. Chinese Journal of Computers, 2010, 33(2):345-355.

[4] C.D. Jensen. P.O. Connell. Trust-based route selection in dynamic source routing[C]. The 4th International Conference, iTrust, Pisa, Italy, 2006.

[5] QU Da-peng, WANG Xing-wei, HUANG Min. Selfish node detection and incentive mechanism in mobile P2P networks[J]. Journal of Software, 2013,24(4):887-899

[6] J. Manickam, L. Martin, S. Shanmugavel. Fuzzy based trusted ad hoc on-demand distance vector routing protocol for MANET[C]. Advanced Computing and Communications, Guwahati, Assam, 2007. 
[7] Leonard Barolli, Evjola Spaho, Fatos Xhafa,et al. Performance evaluation of an integrated fuzzy-based trustworthiness system for P2P communications in JXTAoverlay[J]. Neurocomp- uting , 2013, 122:43-49.

[8] Zhong S, Wu F. On designing collusion resistant routing schemes for non-cooperative wireless ad hoc networks[C]// Proc. Of the 13th Annual ACM Int'l Conf. on Mobile Computing and Networking (MobiCom). Montreal: ACM Press, 2007. 278-289.

[9] Wang Jun. The research of wireless sensor network energy effectiveness [D]. Nanjing: Department of Computer Science and Technology, Nanjing University, 2012.59-68

[10] HU Bao-qing. Fuzzy theory basis [M]. V.2. WU HAN: WUHAN UNIVERSITY PRESS, 2010.

[11] Saaty T L. Fundamentals of decision making and priority theory (2nd ed.)[M]. Pittsburgh, PA:RWS Publications, 2000.

[12] LI Yong-Jun, DAI Ya-Fei. Research on trust mechanism for peer-to-peer network [J]. Chinese Journal of Computer, 2010, 33(3): 390-404. 